\title{
Operator Traces and Holography
}

\author{
Michael R. Douglas
}

\begin{abstract}
We describe a version of AdS/CFT duality proposed by Sezgin, Sundell, Klebanov and Polyakov, between the $O(N)$ model in two dimensions and Fradkin-Vasiliev higher spin gravity in three dimensions, and begin to reformulate it as a mathematical conjecture expressing traces of differential and pseudodifferential operators in terms of a classical boundary value problem in an infinite-component Chern-Simons theory.
\end{abstract}

\section{Contents}

1. Introduction 132

2. Dualities between quantum field theory and gravity 135

2.1. Matrix model/noncritical string duality 137

2.2. AdS/CFT and the conformal boundary 138

2.3. Global symmetries and gauge symmetries 140

3. Free field-higher spin gravity duality 142

3.1. Higher spin gravity 143

3.2. Duality between correlation functions 145

4. Why should gauge-gravity duality work? 146

4.1. $\mathrm{AdS}_{1} / \mathrm{CFT}_{0}$ duality 147

4.2. Determinants of operators 150

4.3. Higher derivative operators 151

4.4. Multiplicative anomaly 153

4.5. Relation to the RG 154

5. Chern-Simons gravity and generalized Liouville theory 155

5.1. Liouville and Chern-Simons gravity 155

5.2. Generalized Liouville theory 157

$\begin{array}{ll}\text { References } & 159\end{array}$

Dedicated to Is Singer on the occasion of his 85th birthday.

(C)2011 International Press 


\section{Introduction}

One of the most striking and unexpected discoveries of the 1994-98 "second superstring revolution" was the AdS/CFT correspondence $[\mathbf{3 3}, \mathbf{4 1}, \mathbf{6 5}$ ], according to which $N=4$ supersymmetric Yang-Mills theory in four dimensions is dual to type IIb superstring theory on $A d S_{5} \times S^{5}$. This finally made precise the physics intuition, developed in the 1970's by 't Hooft, Polyakov, Migdal, Witten and others $[\mathbf{4 9}, \mathbf{5 8}]$, that a four-dimensional gauge theory should have a string description in the large $N$ limit. Since then, the correspondence has been much generalized [1] and has found many applications, especially in providing simple solvable models exhibiting nonperturbative physical phenomena such as confinement, dissipation and quantum phase transitions $[\mathbf{5 6}]$.

However, despite a good deal of work, the microscopic workings of the duality are still not well understood. Various ideas have been developed, such as the holographic renormalization group [18], and integrability [44], which has led to very impressive computations and comparisons at finite gauge coupling $[\mathbf{9}, \mathbf{3 1}]$. But many basic questions, such as the class of field theories with gravity duals, or the mapping between the variables on the two sides, seem to not yet have precise answers.

Although dualities can be understood in depth in simple models [16], usually the complexity of the two theories being related puts most precise statements out of reach. For example, just to define the $N=4$ super Yang-Mills correlation functions requires renormalization - although the beta function is zero, all nontrivial gauge invariant operators are composite operators, which must be renormalized. The usual way this renormalization is phrased in physics requires making choices, at the very least a basis for Hilbert space, and usually much more structure. This dependence plausibly corresponds to some scheme dependence in the dual superstring theory, including and generalizing coordinate transformations, field redefinitions, and perhaps other symmetries. But to make the duality precise for correlation functions of generic operators, one must get these prescriptions to match, which is a formidable technical problem.

Another symptom of this complexity is that, compared to other topics in string theory, so far AdS/CFT has inspired relatively few mathematical developments. While there are some very interesting works along these lines, such as $[\mathbf{4 2 , 6 6}]$, more typically what is discussed is rather intricate, and often simpler to understand in other terms. From this point of view, we should start with simpler versions of the duality, still involving field theory in higher dimensions, to make more fundamental contact with mathematics.

Probably the simplest field theory-gravity duality, after the "old matrix models" $[\mathbf{2 2}]$ which are dual to linear dilaton backgrounds rather than AdS, is the conjecture of Sezgin and Sundell [53] and Klebanov and Polyakov [38], which states that the so-called $O(N)$ model in $D=3$ dimensions is dual to a $D+1=4$ dimensional theory of higher spin gravity developed by Fradkin 
and Vasiliev, and many others [59]. Although less discussed, both the $O(N)$ models and higher spin gravity make sense in arbitrary dimension $D[60]$, and one can make analogous duality conjectures for any $D$.

The $O(N)$ model is exactly solvable in the large $N$ limit, because (as we review shortly) it is simply related to a quantum theory of $N$ free bosons. This theory has an infinite series of higher spin conserved currents, and by physics arguments we will explain, the dual gravitational theory must have a corresponding series of massless fields with spin greater than two. It turns out to be difficult to find unitary quantum field theories of this type, and the consistency of the Fradkin-Vasiliev type theory is nontrivial. It turns out to make sense only in AdS backgrounds, which is part of the motivation for the duality conjecture.

Since the conjecture was made, it received support from several comparisons of correlation functions, however since there are significant differences with the standard AdS/CFT conjectures (for example, the $O(N)$ model is not a gauge theory) its status remained unclear. But recently, a complete check at cubic order has been done by Giombi and Yin [28], which seems to us fairly compelling evidence.

Physically, although the $O(N)$ model itself is now considered a bit trivial, in principle the duality extends to a much larger class of theories, with arbitrary kinetic terms and thus arbitrary dispersion relations for free bosons or fermions, which are of interest in condensed matter physics. The problem of finding the gravity dual of a free field theory has also attracted attention as a starting point for understanding AdS/CFT in more depth [29].

From a mathematical point of view, the partition function of $N$ free bosons is simply the determinant of a Schrödinger operator (raised to the power $-N / 2$ ) on a $D$-dimensional Riemannian manifold, while its generalization to the generating functional of correlation functions is the determinant of a general multi-parameter family of partial differential operators. Thus, the mathematical statement of this duality is an exact formula for such determinants, ${ }^{1}$ in terms of critical points of an action functional of $D+1$-dimensional fields evaluated with specified boundary conditions, corresponding to the choice of operator. This sounds rather interesting and surprising, as determinants and traces of partial differential operators have been studied very extensively and, at least to the author, no such general mathematical results come to mind.

One goal of the present note is simply to explain this duality in a way that we hope a mathematician can more easily follow. Besides the usual language barriers, which by now are not that major (especially for Is), there are a number of significant differences in how these topics are approached in math and in physics, which need to be overcome to make a satisfactory and precise mathematical statement. We will also try to explain these differences to physics readers.

\footnotetext{
${ }^{1}$ At least for manifolds which can appear as a conformal boundary, such as the sphere.
} 
In section 2 , we briefly review gauge-gravity dualities, their origins in the large $N$ limit, and the development of the gravitational point of view. A particularly important point for us, which was actually discovered before the rest of the picture [14], is the relation between symmetries of the gauge theory, and asymptotic symmetries of gravity. A good mathematical introduction to AdS/CFT is [3], and many points which we skim over are explained in more detail there.

In section 3, we describe the physics duality conjecture relating the $O(N)$ model with Vasiliev theory, and explain why this is essentially equivalent to a duality conjecture involving a free bosonic theory. We then discuss some of the physics background behind the conjecture.

In section 4, we survey a few of the questions we should answer in explaining how such dualities work. We then turn to a simple but so far as we know unexplored special case, that of $D=0$, in which the mathematics reduces to the need to take the inverse and the trace of the logarithm of a matrix. We find that, as one might hope, a simple 1d bulk theory can work in this case. Since the $D \geq 1$ theories involve analogous mathematics, with matrices replaced by differential operators, a reasonable way to proceed might be to carefully make this replacement, which might enable a simple and explicit proof of the duality.

In section 5, we begin to turn this idea into a mathematical conjecture about determinants of families of pseudo-differential operators. While the physics arguments suggest that there will be a similar conjecture in any dimension $D$, here we will only cover the case $D=2$, where it generalizes a known

FACT 1. The logarithm of the determinant of the scalar Laplacian $\Delta$ on a two-dimensional manifold $\Sigma$, as a functional of the metric on $\Sigma$, is equal to the action of a particular solution of an $S L(2, \mathbb{R}) \times S L(2, \mathbb{R})$ ChernSimons theory on a three-dimensional manifold $M$ with boundary $\partial M \cong \Sigma$, with boundary conditions determined by the metric on $\Sigma$.

This follows by combining the Polyakov formula for these determinants $[\mathbf{2}, \mathbf{4 8}]$ with results in $2+1$ gravity $[\mathbf{7}, \mathbf{1 7}, \mathbf{4 3}, \mathbf{5 5}]$. Conceptually, this is an example of a general relationship between anomalies in $D$-dimensional QFT (here the conformal anomaly) and Chern-Simons terms in $D+1$-dimensions.

Similarly, by combining the proposed $O(N)$ model duality with results on higher spin gravity, we make

CONJECTURE 1. The logarithm of the determinant of a pseudodifferential operator $\mathcal{O}$ on a two-dimensional manifold $\Sigma$, or a family of operators $\mathcal{O}(t)$, is equal to the action of a particular solution of an $\mathcal{G} \times \mathcal{G}$ Chern-Simons theory on a three-dimensional manifold $M$ with boundary $\partial M \cong \Sigma$, and boundary conditions determined by the choice of $\mathcal{O}(t)$. Here $\mathcal{G}$ is an infinite dimensional Lie algebra called $h s(1,1)$ and defined in §3.1. 
One realization of $h s(1,1)$ is the subalgebra of area-preserving vector fields on the two-dimensional hyperboloid $a^{2}-b^{2}-c^{2}=1$, which are odd under inversion. To begin explaining why this conjecture extends the theorem, we note that since $S L(2, \mathbb{R})$ acts on this hyperboloid by isometries, we have $S L(2, \mathbb{R}) \subset \mathcal{G}$. Thus, the case $\mathcal{O}(t)=\Delta$ corresponds to a subset of boundary conditions in which the $\mathcal{G} \times \mathcal{G}$ solution actually lives in $S L(2, \mathbb{R}) \times$ $S L(2, \mathbb{R})$. Of course, we will need to say far more to motivate a conjecture of this generality.

It is a pleasure to thank Luca Mazzucato, Leonardo Rastelli and Shlomo Razamat for discussions on these topics, and Is Singer for encouraging me over the years to think more mathematically about large $N$ limits.

\section{Dualities between quantum field theory and gravity}

Let us start by reviewing the physical framework of large $N$ limits. We begin by defining a quantum field theory partition function $Z_{Q F T, N}$ for a sequence of QFTs labelled by $N \in \mathbb{Z}^{+}$, schematically

$$
Z_{Q F T, N}=\int[d \Phi] e^{-N^{2} \sum_{i} t^{i} O_{i}[\Phi]}
$$

Here $(\partial M, \gamma)$ is a manifold with Riemannian metric $\gamma$, and $\Phi$ is a map from $\partial M$ to a 'configuration space' - this could be a $U(N)$ connection, a map to a vector space with a linear $U(N)$ action, a direct sum of these, perhaps with some fermionic (odd) variables, and so on. We denote the space of such maps as $\mathcal{C}_{N}$. Thus, for $\operatorname{dim} M \geq 1$, the integral $\int_{\mathcal{C}_{N}}[d \Phi]$ is a functional integral, requiring regularization, renormalization, and the whole works.

The $O_{i}$ are real or complex valued functionals on $\mathcal{C}_{N}$, usually called 'observables' or 'operators.' They appear in Eq. (1) weighed by parameters (or 'couplings') $t^{i}$. One way to think about this is to regard $Z_{Q F T, N}$ as a generating function for expectation values under the integral, as in

$$
\frac{\partial}{\partial t^{i}} \log Z_{Q F T, N}=\mathbb{E}\left[O_{i}\right] \equiv\left\langle O_{i}\right\rangle
$$

and analogous $n$ 'th order derivatives, the $n$-point correlation functions.

To some extent, the metric $\gamma$ can also be thought of as one of the parameters $t$, and its corresponding observable $O_{\gamma}$ is called the 'stress-energy tensor.' However, in the standard discussions, since almost all of the other observables $O_{i}$ depend on the metric, typically in a nonlinear way, the metric dependence is more complicated than that suggested by Eq. (2). And this is the tip of an iceberg, as once one gets into regularization and renormalization, the exponentiated linear dependence on the $t^{i}$ which we wrote in Eq. (1) turns out to be a great oversimplification.

The defining property of the sequences of QFTs we consider is that the $\mathcal{C}_{N}$ have a uniform definition in terms of a space $\mathcal{C}$ of maps into an algebra $\mathcal{A}$, or related structures such as a module for $\mathcal{A}$ and a connection over it. We 
will also need a trace on $\mathcal{A}$, and might also need an involution on $\mathcal{A}$ or other structure. The concrete physics model $Z_{Q F T, N}$ is then obtained by taking $\mathcal{A}=\operatorname{Mat}_{N} \equiv \operatorname{Hom}\left(\mathbb{C}^{N}, \mathbb{C}^{N}\right)$. Thus $\mathcal{C}_{N}$ admits an action of $U(N)$ inherited from the linear action on $\mathbb{C}^{N}$. We require that this is a symmetry; in other words $U(N)$ acts on $\mathcal{C}_{N}$ in a way which preserves the measure $[d \Phi]$ and the observables $O_{i}$.

Although normally one discusses gauge theories in which $\mathcal{C}$ includes a connection over $\partial M$, the focus in this paper will be on the simpler case $\mathcal{C}_{N} \cong \operatorname{Map}\left(\partial M, \mathbb{C}^{N}\right)$, in more abstract terms $\mathcal{C}$ is a module for $\mathcal{A}$.

Having defined the sequence of QFTs, a basic claim about the $N \rightarrow \infty$ limit is

Claim 1. ('t Hooft, Witten): There is a list of observables $O_{i}$ which have good limits, obtained by taking a normalized trace of elements of $\mathcal{A}$, such that the limiting partition function is a sum over saddle points in the following sense:

$$
\lim _{N \rightarrow \infty} Z_{Q F T, N}[\partial M, \gamma, t]=\left.\lim _{N \rightarrow \infty} e^{N^{2} S_{Q G}\left[\partial M, \gamma, O_{i}\right]}\right|_{\partial S_{Q G} / \partial O_{i}=0} .
$$

In words, the large $N$ limit of the logarithm of $Z_{Q F T}$ (the free energy or effective action) is given by evaluating a functional $S_{Q G}$, which is universal for a particular QFT, at its critical point(s) $d S_{Q G}=0$.

Let us sketch a proof of this in the simplest case, of a matrix integral, following [12]. We take $\partial M$ to be a point, and $\mathcal{C}$ to be the hermitian elements $\Phi=\Phi^{*}$ of $\mathcal{A}$. We then consider the integral

$$
Z_{M M, N}[g]=\int_{\mathcal{C}}[d \Phi] e^{-N \sum_{i \geq 1} t_{i} \operatorname{Tr} \Phi^{i}}
$$

where $[d \Phi]$ is the product of Lebesgue measure for each matrix component. Since the integrand is invariant under the conjugation $\Phi \rightarrow U^{\dagger} \Phi U$ with $U \in$ $U(N)$, it is a function of the $N$ eigenvalues of $\Phi$, or equivalently the first $N$ moments $\operatorname{Tr} \Phi^{i}$.

One can then change variables in the integral from the components of $\Phi$, to a choice of group element $U \in U(N)$ and a choice of moments. This brings in a Jacobian, which is the volume of a $U(N)$ orbit with specified moments. It has a simple closed form expression, which can be found in [12].

To heuristically justify the large $N$ limit, we make the following two observations. First, for a 'typical' orbit in which $\Phi$ has order 1 eigenvalues, the moments $\operatorname{Tr} \Phi^{i} \propto N$, so the exponent in Eq. (6) is of order $N^{2}$. Thus we define the observables

$$
O_{i} \equiv \frac{1}{N} \operatorname{Tr} \Phi^{i}
$$


and write

$$
Z_{M M, N}[g]=\int \prod_{i} d O_{i} \operatorname{Vol}\left(O_{i}\right) e^{-N^{2} \sum_{i \geq 1} t^{i} O_{i}}
$$

where $\operatorname{Vol}\left(O_{i}\right)$ is the volume of a $U(N)$ orbit. Then, the volume of a typical orbit has the large $N$ asymptotics

$$
\operatorname{Vol}\left(O_{i}\right) \sim \exp N^{2} S_{e f f}\left(O_{i}\right),
$$

in terms of an 'entropy functional' $S_{\text {eff }}$, the 'free entropy' of Voiculescu [61]. Thus, in the limit, Eq. (6) is dominated by its saddle points, and we recover Eq. (3) with

$$
S_{S G}=S_{e f f}\left(O_{i}\right)-\sum_{i} t^{i} O_{i}
$$

It is natural to try to explain this by finding an algebra with trace $\mathcal{A}$, such that the values of $O_{i}$ at the saddle point are obtained by evaluating Eq. (5) for a particular $\Phi \in \mathcal{A}$, the 'master field.' In this example, this can be done using the free probability theory of Voiculescu [62]. Equally explicit treatments of the limit have been made for other simple quantum theories, such as matrix quantum mechanics, and $D=2$ Yang-Mills [54]. It is tempting to believe that someday this will be made precise for more complicated quantum field theories. There are also probabilistic definitions of the limit of the integral Eq. (6), based on large deviation principles [34].

2.1. Matrix model/noncritical string duality. So far, while we have motivated the claim of Eq. (3), we have not explained why $S_{S G}$ should have any relation to string theory or quantum gravity in $D+1$ dimensions. Let us briefly recall how this works in the simpler matrix model examples, developed in the late 1980's. In these theories, the relation to string theory is precisely the one postulated by 't Hooft, that a planar diagram is a discrete approximation to a string world-sheet, and that string theory is obtained by taking a continuum limit.

The simplest example or "pure gravity" is defined in terms of the matrix integral Eq. (4) as a "double scaling limit" $[\mathbf{1 3 , 2 3 , 3 2}]$ taking the couplings $t^{i}$ to a critical point as $N \rightarrow \infty$. Here $D=0$ and the dual theory is the so-called "c $=0$ string," a non-critical bosonic string with a one-dimensional target space. In terms of the $\Phi$ variable, one formulates observables depending on a new coordinate $r$, as

$$
O_{r} \equiv \operatorname{Tr} e^{-r \Phi}
$$

or some functional transform of this, and rewrites Eq. (8) in terms of these. The coordinate $r$ then parameterizes a one dimensional manifold $M \cong \mathbb{R}^{+}$, whose boundary at $r=0$ might be identified with the point $\partial M$. 
The idea that the $D=1$ theory should be geometric and thus is some theory of gravity is motivated by the general arguments that closed string theory contains gravity. Of course, readers who have heard that bosonic string theory requires $D=26$ may be wondering how a one dimensional string theory can make sense. The answer $[49]$ is that one can turn on a world-sheet coupling which shifts the conformal central charge. Such a coupling arises naturally upon considering a two-dimensional world-sheet metric, so that the new coordinate $r$ then corresponds (loosely) to a distance scale on the world-sheet. This suggests that fixing the couplings $t$ to local observables on the world-sheet, corresponds to putting boundary conditions at $r \rightarrow 0$, leading to a string theory space-time picture.

This picture became clearer in the " $c=1$ model," for which the QFT is the $D=1$ quantum mechanics of a hermitian matrix $M$, with a potential $V=-\operatorname{tr} \Phi^{2}$. Now we can take the field theoretic dimension to be 'time' and the extra $r$ dimension of Eq. (9) to be 'space,' so that the dual $D+1=2$ dimensional theory naturally lives on a Lorentzian space-time. In this case, the equations of motion $d S_{S G}=0$ following from the counterpart of Eq. (8) are a second order hyperbolic PDE $[\mathbf{1 9}, \mathbf{4 7}]$, one can develop a scattering theory, according to which waves (small perturbations) sent in at $r \sim 0$ enter the system, bounce off a 'wall' at large $r$, and come back to $r \sim 0$, defining an S-matrix. Again, Eq. (3) holds with a QFT coupling-boundary data relation.

The heuristic physics explanation for this relation is the 'UV/IR correspondence,' according to which the extra dimension $r$ in the quantum gravity corresponds to a 'renormalization group scale' parameter, here acting on the world-sheet. The boundary condition corresponds to fixing the QFT action at short distances (the UV), while the evolution equation $d S_{S G}=0$ somehow corresponds to incorporating quantum fluctuations at larger distances. Finally, the large $r$ boundary condition or 'wall' is a regularity condition, corresponding to the fact that the renormalization group removes (and does not create) degrees of freedom. Thus many elements of gauge-gravity duality were visible in these models; on the other hand supersymmetry played no role, and the extra dimension looked very different from the field theory dimensions, giving little guidance for how to go to $D>1$.

2.2. AdS/CFT and the conformal boundary. Before AdS/CFT, the search for string duals of gauge theory had generally focused on modifying the world-sheet action for the string, to avoid the following paradox: In quantum field theory, local observables, for example a correlation function of a set of operators at distinct points, are easy to define. On the other hand, in quantum gravity, diffeomorphism invariant local observables are nearly impossible to define, because they are highly nonlinear in the metric, a fluctuating quantum variable. And in string theory, the extended nature of the string makes this problem even worse. 
The now accepted solution of this paradox seems to have first appeared in [50], and consists of taking the string to propagate in a $D+1$-dimensional metric of the form

$$
d s^{2}=\frac{\ell^{2}}{r^{2}}\left(d r^{2}+\gamma\right)
$$

where $r$ is an extra 'radial' coordinate, and $\ell$ is a (fixed) curvature length. In words, we introduce a family of space-time metrics parameterized by an extra coordinate $r$, related by an overall rescaling. For $\gamma$ the Minkowski metric on space-time, the metric Eq. (10) covers part of a constant negative curvature metric with isometry group $S O(D, 2)$, the anti-de Sitter (AdS) space-time.

The metric Eq. (10) first appeared this way in string theory by looking at the geometry near the horizon of a group of D3-branes; it is the direct product of Eq. (10) with a round $S^{5}$. Since the D3-branes can also be described (at low energies) by $N=4$ super Yang-Mills theory, there is a direct physical argument for the duality in this case [41]. The relation of correlation functions to boundary conditions was then proposed in $[\mathbf{3 3}, \mathbf{6 5}]$.

$N=4$ super Yang-Mills, here denoted $\mathrm{MSYM}_{4}$, is a $D=4$ Yang-Mills theory with various scalar and fermionic fields and an action chosen to realize the maximal supersymmetry possible in $D=4$ in a non-gravitational theory. By doing quantum perturbation theory, one learns that it is a superconformal field theory, so the Yang-Mills coupling is a parameter of the quantum theory. Doing the functional integral over fields which live on a $D$ dimensional Riemannian manifold $(\partial M, \gamma)$, one obtains $Z_{Q F T, N}$ of Eq. (3).

The right-hand side of Eq. (3), at finite $N$, is a quantum gravity or string theory partition function. For MSYM, it is type IIb superstring theory compactified on $S^{5}$, to get a $D+1=5$-dimensional quantum gravity. Heuristically, one also thinks of this partition function as defined by a functional integral, now over a fluctuating metric $g$ and various fields $\phi$. While this intuition has never been made precise for quantum gravity or string theory, even to physics standards, in Eq. (3) we sidestep this issue by arguing that in the large $N$ limit, the quantum gravity partition function reduces to a sum over critical points of the supergravity action $S_{Q G}$. Thus, granting the picture of the previous subsection, we obtain a concrete form of Eq. (3).

Now, to explain the solution of the paradox, let us grant that the problem with local observables in quantum gravity only arises when we try to localize on length scales shorter than some fixed length scale $L$, which characterizes the fluctuations of the metric (the Planck scale), the size of a string (the string scale), or other quantum gravity fluctuations. This is physically reasonable: after all, despite the underlying quantum nature of gravity, experimental physicists can work with very short distances $d$ using the ordinary classical picture of space-time, because $L \sim 10^{-33}$ or so (the Planck scale in our universe) and $d \gg L$. 
Applying these ideas to quantum gravity in AdS, since $L$ is a physical length scale, it relates to distances defined using the metric Eq. (10). But, a fixed space-time distance $d$ measured in the metric $\gamma$ corresponds to an AdS metric distance $d / r$, which as $r \rightarrow 0$ becomes much greater than $L$. Thus, by associating QFT correlation functions with boundary conditions at $r \rightarrow 0$, we can localize them on arbitrarily short scales $d$, and avoid the paradox.

It is convenient that the metric Eq. (10) is conformal to a metric $\tilde{d} s^{2}=$ $r^{2} d s^{2}$ which is non-singular at $r=0$. Indeed, since $r=0$ is at finite distance in this metric, we can think of it as the boundary $\partial M$ of a closed manifold with metric $(M, \tilde{g})$. In mathematical terms, $(M, \tilde{g})$ is the conformal compactification of $(M, g)$. In this way, we can replace the physics idea of scattering boundary conditions at $r \sim 0$, with local boundary conditions on $\partial M$. Thus we have taken another step towards making Eq. (3) precise.

A familiar variation is to take $\gamma$ with Riemannian signature, defining a 'statistical field theory.' This corresponds to Riemannian $\gamma$ in Eq. (10). For example, taking $(\partial M, \gamma)$ to be the round $D$-sphere, the metric Eq. (10) will be $D+1$-dimensional hyperbolic space.

One then expects the equations $d S_{S G}=0$ to be elliptic, and the corresponding boundary value problem to have a unique solution. For example, a metric on $M$ satisfying the Einstein equation with negative cosmological constant (constant negative Ricci curvature), should be determined by the conformal class of its restriction to $\partial M$. As discussed in [3], the nonlinearity of the Einstein equations makes such a claim highly nontrivial, and false without restrictive assumptions. But, as shown by Fefferman and Graham [27], it is generally true in the sense of an asymptotic expansion: given $\gamma$ on $\partial M$, one can solve for the higher order terms $\gamma^{(k)}$ in

$$
d s^{2}=\frac{\ell^{2}}{r^{2}}\left(d r^{2}+\gamma+r^{2} \gamma^{(2)}+\cdots\right),
$$

if $D$ is odd to all orders. This can be used to define correlation functions of the metric as in Eq. (2) (with $t \rightarrow \gamma$ ).

A simpler example is a scalar field $\phi$ whose equation of motion $\partial S_{S G} / \partial \phi=0$ is simply the Laplace equation on $M$. By taking Dirichlet boundary conditions, one gets a well posed problem, whose solution is given by a "boundary-to-bulk Green function,"

$$
\phi(x, r)=\int_{\partial M} d^{D} x^{\prime} \phi\left(x^{\prime}, 0\right) G\left(x^{\prime} ; x, r\right) .
$$

One can then treat nonlinear terms in $\partial S_{S G} / \partial \phi$ by the usual perturbative approach. Since one only needs a classical solution, the series expansion for an $n$-point function will have a finite number of terms, and there are many such calculations for low $n$ in the literature.

2.3. Global symmetries and gauge symmetries. It is rather magical that the large $N$ action $S_{S G}$ of Eq. (3), which according to the arguments 
so far might be too complicated or abstract to work with, ever takes such a simple and explicit form as a supergravity action. While this follows from the string theory intuition, a priori this argument is very closely tied to the specific theories which come out of string and $M$ theory, leaving the question of what class of QFTs might admit gravity duals completely open. Is it only these theories, or theories which can be obtained from these by adding operators? If it is a larger class, what characterizes it - gauge symmetry? conformal invariance? supersymmetry? In some cases such as $\mathrm{MSYM}_{4}$, one has integrability. Each of these properties have been suggested to be important.

Perhaps the best clue we have at present is the relation between global symmetries of the QFT, and gauge symmetries of the gravity theory. For example, $\mathrm{MSYM}_{4}$ has an $S U(4)$ R-symmetry (a global symmetry under which the supercharges transform nontrivially), while IIb supergravity compactified on $S^{5}$ leads to a five-dimensional gauged supergravity with $S U(4)$ gauge fields. The most far reaching case is the relation between the stresstensor of QFT, a spin two operator which generates diffeomorphisms acting on $(\partial M, \gamma)$, and the metric $g$ of the dual gravity theory. Clearly this relation lies at the heart of the matter.

There are various physical arguments for it. In the context of theories which can be obtained from branes in string theory, such as MSYM 4 and D3branes, one has the general comment that any gauge symmetry of the string theory, must couple to some current (operator) on the brane which generates a corresponding global symmetry [8]. One also has a general relation between global anomalies on the brane, and corresponding Chern-Simons terms in the bulk $[\mathbf{3 0}, \mathbf{6 5}]$.

Granting the gauge-gravity duality, one can reverse this logic by observing that global symmetries of QFT are generated by operators (currents) with spin (i.e., which transform nontrivially under the Lorentz group). Such an operator will correspond to a massless field with spin in the bulk theory. But massless fields with spin only make sense in a unitary quantum theory if there is a corresponding gauge invariance. Without gauge invariance, the Hilbert space for a particle with spin would decompose into finite dimensional linear representations of the Lorentz group, but this is noncompact and has no finite dimensional unitary representations. This paradox is evaded by embedding the Lorentz group into a larger group, whose representations can be unitary after quotienting by the gauge group (this is usually done by BRST quantization, i.e. taking equivariant cohomology). The same logic can be applied to translational symmetry, which is generated by a spin two current (the stress tensor), to explain the need for general covariance and derive the Einstein-Hilbert action [21].

A final reversal of this argument would be to derive the global symmetry of the QFT from the bulk theory, purely in the context of gauge-gravity duality (as opposed to string theory or branes). In fact such an argument in a sense precedes the others, as it amounts to deriving the group of symmetries 
which act on the asymptotic region of a space-time in gravity. This is an old question: for example, how does one see geometrically that the Poincaré group acts on an asymptotically flat solution, without referring to explicit coordinates? How does one define the corresponding conserved quantities? These are subtle problems, about which entire reviews have been written [57], and are clearly central to this subject.

Recent works on the asymptotic symmetries of higher spin gravity include $[\mathbf{1 5}, \mathbf{3 6}]$.

\section{Free field-higher spin gravity duality}

Although the usual discussion involves gauge theory, there is a simpler and older class of field theories with large $N$ limits, the so-called vector models. The basic example is a theory of $N$ complex scalar fields denoted $\vec{\psi}$, with the action

$$
S_{f t}=\int_{\partial M} \sqrt{\gamma} \gamma^{i j} \partial_{i} \vec{\psi} \cdot \partial_{j} \vec{\psi}+t|\vec{\psi}|^{2}+\lambda\left(|\vec{\psi}|^{2}\right)^{2} .
$$

Here $\vec{a} \cdot \vec{b}$ is the usual hermitian inner product on $\mathbb{C}^{N},|\vec{a}|^{2}=\vec{a} \cdot \vec{a}, \lambda \in \mathbb{R}$, and in addition we allow a real-valued function $t: M \rightarrow \mathbb{R}$ (in physics terms, a position-dependent mass).

It is not hard to obtain a large $N$ action of the form Eq. (8) for this theory, as done for example in [20]. It is also not hard to compute the partition function directly. Let us start with the special case $\lambda=0$. Since the action is then quadratic in $\vec{\psi}$, a straightforward application of the theory of Gaussian functional integrals tells us that

$$
\begin{aligned}
Z_{Q F T, N}[\gamma, t] \equiv e^{-N F[g]} & =\operatorname{det}\left(\Delta_{\gamma}+t\right)^{-N} \\
& =\exp -N \operatorname{Tr} \log \left(\Delta_{\gamma}+t\right),
\end{aligned}
$$

where $\Delta_{\gamma}=-\gamma^{i j} \nabla_{i} \nabla_{j}$ is the scalar Laplacian. This depends on $N$, but in a trivial way.

Of course, this will require regularization to make sense. We could take det to be the zeta function regularized determinant [51].

$$
\operatorname{det} A=\exp \lim _{s \rightarrow 0} \frac{\partial}{\partial s} \operatorname{Tr} A^{s}
$$

We will return to this point in $\S 4.4$.

Having warmed up with $\lambda=0$, to handle the general case, we note that

$$
\int d t e^{\frac{t^{2}}{2 \lambda}+t|\vec{\psi}|^{2}}=\sqrt{2 \pi \lambda} e^{-\lambda\left(\left.\vec{\psi}\right|^{2}\right)^{2}}
$$

Thus, we can eliminate the quartic term in Eq. (13) in favor of a quadratic term, at the cost of doing another functional integral over $t$. If we take 
$\lambda \propto 1 / N$, this integral becomes

$$
\begin{aligned}
\int d t e^{\frac{N t^{2}}{2 \lambda}} Z_{Q F T, N}[\gamma, t] \\
=\int d t e^{N\left[\frac{t^{2}}{2 \lambda}+\operatorname{Tr} \log \left(\Delta_{\gamma}+t\right)\right]}
\end{aligned}
$$

and in the large $N$ limit, it will be dominated by a saddle point. Thus, the case $\lambda \neq 0$ can also be solved in the large $N$ limit, if we can get a sufficiently explicit expression for Eq. (14).

In the usual physics case of $\gamma$ the Euclidean metric and constant $\lambda$, it is easy to get such an explicit expression by diagonalizing $\Delta_{\gamma}$, and thus the $O(N)$ model is exactly solvable. However, if we consider more general metrics, or position-dependent $t$, this is not so easy. One can of course treat the case of perturbations around this solvable case, but an explicit formula for Eq. (14) is not known.

Since the relation between Eq. (13) and Eq. (14) was simply that of Gaussian functional integration, at least formally it generalizes to an arbitrary linear operator in the action,

$$
\int d \psi e^{-\vec{\psi} \psi, \mathcal{O} \vec{\psi})}=(\text { const. }) \exp -N \operatorname{Tr} \log \mathcal{O},
$$

with

$$
\mathcal{O}=t+\gamma_{1}^{i} \partial_{i}+\gamma_{2}^{i j} \partial_{i} \partial_{j}+\gamma_{3}^{i j k} \partial_{i} \partial_{j} \partial_{k}+\cdots
$$

We could treat this expansion in two ways. One is to try to define a functional of all of these coefficients, say $\operatorname{Tr} \log \mathcal{O}\left[t, \gamma_{1}, \gamma_{2}, \ldots\right]$. Less ambitiously, we could regard Eq. (19) as the generating functional of correlation functions of general local operators, obtained by taking derivatives with respect to the $\gamma_{n}$ at $\gamma=0$. This allows us to make contact with computations such as that of $[\mathbf{2 8}]$, so for this section let us be satisfied with this definition.

3.1. Higher spin gravity. Although there are several approaches to higher spin gravity, perhaps the simplest is to define it as a gauge theory, along the general lines of Ashtekar's approach to $3+1$ gravity [4] and Witten's approach to $2+1$ gravity [64], but with an infinite dimensional gauge group. In general terms, instead of the basic field being the metric tensor, one takes as fields the frame (or $D$-bein) and spin connection, and reinterprets these as components of a gauge connection. Just as it turns out that the Einstein-Hilbert action can be re-expressed as a gauge theory action, so too can the higher spin gravity action.

Thus, let us first review the analogous formulation of standard $2+1$ dimensional gravity. In these dimensions, the simplest gauge theory action is the Chern-Simons action, and Einstein gravity was reformulated in these terms in [64]. One starts with a three dimensional manifold $M$, and a vector 
bundle $V$ over $M$ with structure group $S O(2,1)$. One then introduces a frame $e \in \operatorname{Hom}(T M, V)$ and a connection $\omega$ on $V$. One then embeds $S O(2,1)$ in a larger group $G$ whose additional generators correspond to translations, and identifies $(e, \omega)$ with components of a $G$-connection. Chern-Simons gravity is then simply Chern-Simons theory on $M$ with group $G$, so a classical solution is a flat $G$ connection.

For $2+1$ gravity with a negative cosmological constant, so that a solution has constant negative Ricci curvature $-\ell^{2}$, one takes $G \cong S L(2, \mathbb{R}) \times$ $S L(2, \mathbb{R})$, with connections $A$ and $\tilde{A}$ and the identification

$$
A=\omega+\frac{1}{\ell} e ; \quad \tilde{A}=\omega-\frac{1}{\ell} e .
$$

The action is the difference of two Chern-Simons actions,

$$
S_{Q G}=S_{C S}[A]-S_{C S}[\tilde{A}] ; \quad S_{C S}[A]=\operatorname{tr} \int A d A+\frac{2}{3} A^{3} .
$$

Its simplest classical solution is the group manifold of $S L(2, \mathbb{R})$ itself, taking its left- and right-invariant connections as $A$ and $\tilde{A}$ respectively. Its universal cover is $\mathrm{AdS}_{3}$. It is conformally compact, with boundary $\partial M \cong \mathbb{R} \times S^{1}$ carrying the flat (Minkowski) metric. The duality conjecture Eq. (3) thus relates this gravitational theory on $\mathrm{AdS}_{3}$ to a two-dimensional QFT on the cylinder.

The formulation of higher spin gravity in these terms is attributed in [36] to [10]. We again take the action Eq. (22), but $A$ and $\tilde{A}$ are now connections on $M$ each taking values in the "higher spin algebra $h s(1,1)$," an infinite dimensional Lie algebra containing an $\operatorname{sl}(2, \mathbb{R})$ subalgebra. Thus, one again has $\mathrm{AdS}_{3}$ as a solution, by embedding the previous connection into this larger algebra.

There are various definitions of $h s(1,1)$ in the physics literature, almost all in terms of generators and relations, or else oscillators. In [11], it is identified with the odd area preserving vector fields acting on a $2 \mathrm{~d}$ hyperboloid with $\operatorname{sl}(2, \mathbb{R})$ symmetry (i.e., the hypersurface $a^{2}-b^{2}-c^{2}=1$ in $\mathbb{R}^{3}$, and 'odd' means odd under inversion).

Another suggested geometric interpretation of $h s(1,1) \oplus h s(1,1)$, and its generalizations to arbitrary $D$, is the Lie algebra contained in the associative algebra of symmetries of the Laplacian.

Definition 1. (Eastwood, [25]) A symmetry of the Laplacian $\Delta$ is a linear differential operator $\mathcal{D}$ such that

$$
\Delta \mathcal{D}=\delta \Delta
$$

for some linear differential operator $\delta$. The algebra of symmetries of the Laplacian is the algebra of such operators with the natural product, with the 
equivalence relation

$$
\mathcal{D} \cong \mathcal{D}+\mathcal{P} \Delta
$$

with $\mathcal{P}$ a linear differential operator.

Eastwood then argues that symmetries of the Laplacian correspond to conformal Killing tensors, which are also the natural symmetries which arise from the point of view discussed in $\S 2.3$. In the particular case of the Laplacian on $\mathbb{R}^{D}$ (with the Euclidean metric), their algebra is the universal enveloping algebra of $S O(D+1,1)$, modulo a two-sided ideal generated by the Killing form (schematically $<V, W>\sim 1$ ). The relation to the algebras used in defining higher spin gravity is discussed in [60].

For present purposes, however, let us simply cite a concrete definition of $h s(1,1)$. It is the space of even polynomials in two variables $\xi^{1}, \xi^{2}$ modulo constants, with the bracket derived from the associative product

$$
\left.(f * g)(\xi) \equiv \exp i\left(\frac{\partial}{\partial \xi^{1}} \frac{\partial}{\partial \eta^{2}}-\frac{\partial}{\partial \xi^{2}} \frac{\partial}{\partial \eta^{1}}\right) f(\xi) g(\eta)\right|_{\xi=\eta} .
$$

The quadratic polynomials generate an $\operatorname{sl}(2, \mathbb{R})$ subalgebra, say

$$
J^{+} \equiv \xi^{1} \xi^{i} ; \quad J^{3} \equiv \xi^{1} \xi^{2} ; \quad J^{-} \equiv \xi^{2} \xi^{2} .
$$

3.2. Duality between correlation functions. It is easy to develop a perturbative expansion for Eq. (19), using

$$
\operatorname{Tr} \log (\mathcal{O}+\delta \mathcal{O})=\operatorname{Tr} \log \mathcal{O}+\sum_{n \geq 1} \frac{(-1)^{n}}{n} \operatorname{Tr}\left(\mathcal{O}^{-1} \delta \mathcal{O}\right)^{n}
$$

Let us look at the three-point correlation function, discussed for $D=3$ in Giombi and Yin [28]. We take $z$ to be a coordinate on $\partial M$ and $\mathcal{O}$ to be the Laplacian, so that $\mathcal{O}^{-1}$ is the standard field theoretic Green function. For $\partial M \cong \mathbb{R}^{D}$,

$$
\left\langle\bar{\psi}\left(z_{1}\right) \psi\left(z_{2}\right)\right\rangle \equiv F\left(z_{1}, z_{2}\right)=\text { const. } \cdot\left|z_{1}-z_{2}\right|^{2-D} .
$$

An $n$-point function of operators $\bar{\psi} \mathcal{O}_{i} \psi$ is then a sum of $(n-1)$ ! terms of the form

$$
\mathcal{A}=\int d^{2} z_{1} \ldots d^{2} z_{n} \mathcal{O}_{1} F\left(z_{1}, z_{2}\right) \mathcal{O}_{2} F\left(z_{2}, z_{3}\right) \ldots \mathcal{O}_{n} F\left(z_{n}, z_{1}\right)
$$

For definiteness, consider $D=3$ and $n=3$, with three operators $J_{i}=$ : $\bar{\psi}\left(z_{i}\right) \partial_{i} \psi\left(z_{i}\right)$ :, one gets expressions like

$$
\frac{\partial}{\partial z_{1}^{i_{1}}} \frac{\partial}{\partial z_{2}^{i_{2}}} \frac{\partial}{\partial z_{3}^{i_{3}}} \frac{1}{\left|z_{1}-z_{2}\right|\left|z_{2}-z_{3}\right|\left|z_{3}-z_{1}\right|}+(2 \leftrightarrow 3) .
$$


The dual expression is found by following the approach outlined in $\S 2.2$; one finds

$$
\mathcal{A}_{k_{1}, k_{2}, k_{3}}^{(S G)}\left(z_{1}, z_{2}, z_{3}\right)=\int d^{2} z d r G_{k_{1}}\left(z_{1} ; z, r\right) G_{k_{2}}\left(z_{2} ; z, r\right) G_{k_{3}}\left(z_{3} ; z, r\right)
$$

where $G_{k_{i}}\left(z_{i} ; z, r\right)$ is the bulk-to-boundary Green function Eq. (12) for a massless spin 1 field (Eq. (2.26) in [65]).

At this general level, there is little resemblance between the two expressions. Actually, given a metric on $\partial M$ with enough conformal isometries, such as the standard cases of $\mathbb{R}^{D}$ or $S^{D}$, symmetry forces the three-point functions to have the same functional dependence, so the nontrivial prediction of the duality is the overall normalizations. However this argument quickly peters out for higher point functions, and loses all strength for general metrics and operators $\mathcal{O}$.

The best studied examples come with their own special simplifications. For example, $D=4$ MSYM has a large superconformal symmetry, which forces many relations between correlation functions. In our primary example of $D=2$, one has holomorphic factorization. In the Chern-Simons dual, this is reflected in the form $\mathcal{G} \times \mathcal{G}$ for the gauge group.

There has been a fair amount of work trying to give more general arguments for this equality, which could work for any of the proposed dualities. One of the more interesting ones appears in [29] and involves a relation between the proper time parameterizations

$$
\mathcal{O}^{-1}=\int_{0}^{\infty} d \tau e^{-\tau \mathcal{O}}
$$

for the QFT Green function Eq. (28) and the bulk-to-boundary Green function Eq. (12), motivated by the physics intuition relating the two sides of Eq. (3) to open and closed strings. This argument is quite simple for the three-point function in a symmetric background, but it is not clear how to take it farther.

In any case, the equality of Eq. (30) and Eq. (31), as demonstrated for $D=3$ in [28], looks sufficiently nontrivial and convinces us to take $O(N)$ higher spin gravity duality seriously.

\section{Why should gauge-gravity duality work?}

Most of the work on this question focuses on the $\mathrm{AdS}_{5} / \mathrm{MSYM}_{4}$ duality. This is a very rich subject which we will not try to do justice to here, instead raising a few questions which the author finds interesting.

First, compared to previous ideas about large $N$ limits and Eq. (3), a lot of the power of AdS/CFT comes from the statement that one can take a second limit, of strong (large) Yang-Mills coupling, in which $S_{S G}$ becomes a local field theory, such as type IIb supergravity. 
Now there is a simple physics argument for this - it follows from the mapping of parameters $\left(N, g_{Y M}\right)$ to the dual string theory coupling and string scale $l_{s}$, which translates the limit $g_{Y M} \rightarrow \infty$ into the limit $l_{s} \rightarrow 0$. In this limit, the higher dimension operators of string theory go off to infinite dimension, leaving only the fields of supergravity.

While this argument sounds quite reasonable, it remains surprising from any field theoretic point of view. Are we sure that it is true? After all, there is another scale in the problem, the AdS curvature radius. There exist gravity theories which are nonlocal on this scale - in fact the higher spin gravity we discussed in section 3 is an example. One might entertain a different conjecture that, even at large Yang-Mills coupling, gravity duals can have similar nonlocality.

For the case of $\mathrm{MSYM}_{4}$, results from integrability are believed to show that all non-supergravity operators do in fact go off to infinite dimension at strong coupling, eliminating this loophole. Granting this, for what class of theories does this work? Integrability is very special, and almost all theories of physical interest are not integrable; the principle which makes this work should be more general.

Second, where does the extra dimension come from? The standard intuition involves the renormalization group. While very believable, it seems fair to say that this has not yet been made as precise as one would like. This question is discussed further in $[\mathbf{2 4}]$.

Third, as we commented in the introduction, making a precise statement away from strong coupling probably requires matching up all of the operators on both sides of the duality. How do we organize all the higher derivative operators? This is surely a question of geometry - what geometry underlies the duality?

4.1. $\mathbf{A d S}_{1} / \mathbf{C F T}_{0}$ duality. Let us return to discuss the duality between free QFT and higher spin gravity. The simplest point of view would be that this discussion depends so little on details, that even a schematic expression like Eq. (19) could have a gravity dual. Since readers with some familiarity with higher spin gravity or other detailed physics discussions may be skeptical at this point, let us explore this idea in the simplest possible context.

Thus, let us consider the $D=0$ case, in which the operators $O[t]$ are $N^{\prime} \times N^{\prime}$ hermitian matrices, i.e. elements of the Lie algebra $u\left(N^{\prime}\right)$. The reason we write $N^{\prime}$ is that, in the standard discussions, one restricts attention to gauge theory or at least to $u(N)$ singlet operators, whereas we want to consider general operators. Of course, one can consider the configuration space $\mathbb{C}^{N^{\prime}} \otimes \mathbb{C}^{N}$, and tensor the operator $O[t]$ of Eq. (19) with the identity acting on $\mathbb{C}^{N}$, so in a free theory this is a distinction without a difference. Thus for ease of notation we simply take $\mathbb{C}^{N}$ as the configuration space in the following, and consider general operators. 
Let $e_{a}$ be a basis for $u(N)$ and write

$$
O[t] \equiv \sum_{a} t^{a} e_{a}
$$

then the partition function Eq. (14) becomes

$$
\begin{aligned}
Z[t] & =\int d^{2 N} \vec{\psi} e^{-\bar{\psi} O[t] \psi} \\
& =\exp -\operatorname{Tr} \log O[t] .
\end{aligned}
$$

The observables of Eq. (2) are

$$
u_{a}=\frac{\partial}{\partial t^{a}} \log Z=\operatorname{Tr} O[t]^{-1} e_{a} .
$$

If we think of $t$ and $u$ as matrices, we have $u=t^{-1}$.

Now, one may ask, how can the simple results Eq. (34) and Eq. (35) be obtained by introducing an extra dimension?

Since the $D=0$ theory admits a global symmetry $O[t] \rightarrow g^{-1} O[t] g$, we expect the bulk theory to be a $u(N)$ or even $g l(N)$ gauge theory,. Thus, we introduce a covariant derivative $D_{r}$ with connection $A_{r}$. Of course, the connection can be gauged to zero in one dimension, so this is not the key point.

We take the couplings $t$ to be boundary conditions for a field $g(r)$ which is an adjoint of $u(N)$. Since we know we will need to take the logarithm, we define $\phi(r)$ to be

$$
g=\exp \phi
$$

Let us try postulating the simplest possible second order equation for $\phi$,

$$
\frac{\partial^{2}}{\partial r^{2}} \phi=0
$$

as would follow from the action

$$
S=\int d r\left(g^{-1} D_{r} g\right)^{2}
$$

in the gauge $A_{r}=0$. Its general solution is $\phi=A r+B$. According to the general philosophy of gauge-gravity duality, we should use the couplings $t$ as a Dirichlet boundary condition, in other words as the leading or nonnormalizable solution. Thus we take $A=\log t$. We then, as in the matrix model examples, postulate a wall at $r=1$. At the wall, we impose the boundary condition $\phi(r=1)=0$, so $B=-A$. This is consistent with the variational equation for the gauge connection, which is $\left[\phi^{\prime}, \phi\right]=0$ which forces $[A, B]=0$. Finally, the normalizable mode determining $u$ is $B=-A$, and the corresponding part of $g$ is $\exp -\log t=t^{-1}=u$. 
Thus, it is easy to get the relation Eq. (35) from a $D+1$-dimensional dual theory. Of course, we postulated all of its ingredients, so the significance of this can only be judged by going on to cases with more structure. Still, we are not done with $D=0$ yet, as we need to check that the value of the action on this solution reproduces Eq. (34) in some sense. This is $\operatorname{Tr} A$, but the action Eq. (38) gives us the (divergent) volume $\int d r$ multiplied by $\operatorname{Tr} A^{2}$. Even if we could argue that this divergent term were meaningful, it would not be correct. Thus, we need to add additional terms and fields to the action to cancel it.

Since the additional term is negative, adding it will lead to problems of stability when we go on to higher dimensions. This is a sign that we need to bring features of gravity into the discussion. In particular, the action Eq. (38) does not have $r$-reparameterization invariance.

Thus, we introduce a metric $d s^{2}=e(r) d r^{2}$ and consider the reparameterization invariant action

$$
S=\int d r e^{-1} \operatorname{Tr}\left(g^{-1} D_{r} g\right)^{2}+\frac{e}{\ell^{2}}+\alpha \operatorname{Tr}\left(g^{-1} D_{r} g\right) .
$$

Starting with $\alpha=0$ and taking constant $e$ gauge, then the constraint from varying $e$ determines

$$
\begin{aligned}
e^{2} & =\ell^{2}\left(g^{-1} D_{r} g\right)^{2} \\
& =\ell^{2} \operatorname{Tr} A^{2}
\end{aligned}
$$

given our boundary conditions. Thus, the divergent part of the action is now

$$
S \sim \int d r \frac{e}{\ell^{2}} \sim \int d r \frac{1}{\ell} \sqrt{\operatorname{Tr} A^{2}}
$$

This is also not what we want. In fact, the term $\operatorname{Tr} A$ which would match Eq. (34), would arise from the $\alpha \operatorname{Tr}\left(g^{-1} D_{r} g\right)$ term. Since it is a total derivative, it does not change the equation of motion, so we can add it, but we still need to subtract the term Eq. (42).

To write an action which does this, we drop the $\ell$ term and instead work in first order formalism with a canonical pair of variables $\left(\phi^{\mu}, \Pi_{\nu}\right)$, and write

$$
S=\int d r \operatorname{Tr} \Pi_{\mu} D_{r} \phi^{\mu}+\frac{1}{2} e \eta^{\mu \nu} \operatorname{Tr} \Pi_{\mu} \Pi_{\nu}+\operatorname{Tr} D_{r} \phi^{1} .
$$

with a constant metric $\eta^{\mu \nu}$. Now the equations of motion and constraint are

$$
D_{r} \phi^{\mu}=-e \eta^{\mu \nu} \Pi_{\nu} ; \quad D_{r} \Pi_{\mu}=0 ; \quad \eta^{\mu \nu} \Pi_{\mu} \Pi_{\nu}=0 .
$$

To solve the constraint with $\Pi \neq 0$, there must be more than one component in $(\phi, \Pi)$, and the metric $\eta^{\mu \nu}$ must have indefinite signature, say $\eta^{00}=-1$ and $\eta^{11}=1$. We take one component, say $D_{r} \phi^{1}$ to be $g^{-1} D_{r} g$ as above, thus we control its boundary condition. Then $\Pi_{1}=A$ as above and $\phi^{1}=A r+B$. 
We also need $\operatorname{Tr} \Pi_{0}^{2}=\operatorname{Tr} A^{2}$ so the second sector just cancels the unwanted term in the action. The term $\operatorname{Tr} A$ we want comes out by taking the total derivative term to only see $\phi^{1}$.

While we would not make overly strong claims for the importance of this toy model, it is a very simple illustration of how the dual of a free theory can work. What did the extra dimension buy us? In terms of the $(\phi, \Pi)$ variables, the dynamics is linear, and the transformation $A \rightarrow B=-A$ is rather trivial. It becomes more nontrivial when we formulate the dynamics in terms of $g=e^{\phi}$. The equations of motion are now

$$
\begin{gathered}
g^{-1} D_{r} g=-e \Pi \\
D_{r} \Pi=\left[\Pi,\left(g^{-1} D_{r} g\right)\right] .
\end{gathered}
$$

The second is still solved by constant $\Pi$, while the first gives a local evolution role for $g$, solved (taking $A_{r}=0$ ) by

$$
g=g_{0} \cdot e^{-\int^{r} d r^{\prime} e\left(r^{\prime}\right) \Pi\left(r^{\prime}\right)} .
$$

The "wall" boundary condition $g=1$ then determines $\Pi=\log g_{0}$, and the dynamics propagates it back to the boundary. In higher dimensions, one can hope that the 'wall' boundary condition will emerge in a less artificial way, as a consequence of continuity in the interior of AdS.

Thus, we can take the logarithm of $O[t]$ using a local $1 \mathrm{~d}$ bulk theory. It exhibits two other features which we might look for in higher dimensions. First, the indefinite metric and constraint in Eq. (43) is suggestive of the role of the conformal factor in the Hamiltonian constraint for gravity. Second, the term $\alpha \operatorname{Tr}\left(g^{-1} D_{r} g\right)$ which reproduced the quantum free energy is a onedimensional analog of the Wess-Zumino term of higher dimensions, which will play an important role in section 5 .

4.2. Determinants of operators. From an abstract point of view, linear differential operators are not so different from matrices, so the $D \geq 1$ case could be treated the same way. Several questions present themselves:

- What is the analog of the group $U(N)$ in the $D=0$ discussion? In other words, what are the natural symmetry algebras and groups associated to a space of differential operators acting on a $D$-dimensional manifold $\partial M$ ?

- To define a determinant such as Eq. (14), we need to regulate the theory. This is no surprise and can be done in many ways, but regularization will spoil some of the formal properties used in our arguments, such as cyclicity of the trace $\operatorname{Tr} A B=B A$. Can we either show that all the properties we need are true, or else characterize their anomalies and fix up the arguments to take these into account? 
- Assuming this can be done, and that we can substitute the appropriate analog of $U(N)$ into Eq. (43), can we explicitly write done the resulting effective action in $D+1$ dimensions? if so, will it be local? In any case, why would there be any symmetry relating the extra dimension to the original $D$ dimensions?

The physics word 'local' usually means that one can write the action as an integral over a functional of the fields and finitely many of their derivatives. Unfortunately, this definition becomes meaningless in a theory with an infinite number of fields. Probably, higher spin gravity should not be regarded as local.

Naively, the analog of $U(N)$ in $D \geq 1$ is a group of differential operators, for example the operators $-\Delta+t$ and everything we can get by taking arbitrary combinations of these. Of course, these are unbounded operators and, although we can add and multiply them, our ability to do anything else is severely limited. And since the inverse of a differential operator is not a differential operator, they do not by themselves form a group.

The basic operation in $\S 4.1$ turned out to be Eq. (45), the evolution of $g$ by right action of a semigroup evolving it to the identity. If we can make sense of the semigroup action $\exp -r \Pi$, then a prescription with boundary conditions along the lines we just gave, could naturally lead to the relation $\Pi=\log g$. But while a semigroup action is easier to define mathematically, the need to work with logarithms of differential operators again forces us to generalize our space of operators.

4.3. Higher derivative operators. To turn Eq. (3) into a satisfactory mathematical conjecture, we should also revisit the meaning of operators like: $\bar{\psi} \mathcal{O} \psi$ involving higher derivatives, as in Eq. (20),

$$
\mathcal{O}=t+\gamma_{1}^{i} \partial_{i}+\gamma_{2}^{i j} \partial_{i} \partial_{j}+\gamma_{3}^{i j k} \partial_{i} \partial_{j} \partial_{k}+\cdots
$$

In section 3, we regarded Eq. (1) as a formal power series in the couplings $\gamma_{n}$. Of course, it would be more attractive to think of $\operatorname{Tr} \log \mathcal{O}$ as a welldefined real valued function of the couplings $\gamma_{n}$. However, one cannot sum these Taylor expansions around zero; if one starts with $\gamma_{n}=0$ for $n>n_{\max }$ (say $n_{\max }=2$ as in Eq. (13)) the $\gamma_{n}$ Taylor series is divergent. This is to say that the higher order derivatives are singular perturbations, on general grounds and in QFT because they change the high energy behavior of $\mathcal{O}$ and thus require changing the regularization prescription.

Although one can take various approaches to dealing with this inconvenience, conceptually the cleanest is to put an explicit cutoff into the definition of $\mathcal{O}$. If one takes the couplings $\gamma_{n}$ to be constant, this is easily done in momentum (Fourier transform) space; for example we could take $p^{2} \rightarrow$ $p^{2} \exp -\alpha p^{2}$. One can then add similarly regulated operators $p^{n} \exp -\alpha p^{2}$, to get regular perturbations. The precise details tend to depend on the problem 
at hand, because all such regulators complicate the explicit expressions, and one has to take advantage of specifics to get tractable results.

In any case, this approach requires us to consider operators $\mathcal{O}$ which are non-polynomial in derivatives. In fact, there are many other reasons to do this, which in mathematics led to the development of the theories of pseudo-differential operators (PDOs) and Fourier integral operators. While we cannot provide an introduction here (see e.g. $[\mathbf{5 2}]$ ), the starting point for this is to consider operators $\mathcal{O}(t)$ acting on functions $f: \mathbb{R}^{N} \rightarrow \mathbb{R}$ of the form

$$
(\mathcal{O}(t) f)(x) \equiv \int d^{D} \xi t(x, \xi) \int d^{D} y e^{i(x-y) \cdot \xi} f(y) .
$$

For example, a linear differential operator with constant coefficients is given by taking $t(x, \xi)$ to be a polynomial in $\xi$. However, we do not require $t(x, \xi)$ to be polynomial; rather we impose conditions on how it behaves at infinity, such as $t(x, \xi) \sim \xi^{m}$ for an $m$ 'th order operator (this is not very precise, see [52] for the actual definitions). The operators satisfying this condition then form a linear space denoted $\mathcal{S}^{m}$. One can also define operator classes with logarithmic growth.

One then has multiplication laws such as

$$
\mathcal{O}(t) \mathcal{O}(u)=\mathcal{O}(t * u)
$$

with

$$
\begin{aligned}
(t * u)(x, \xi) & =\frac{1}{(2 \pi)^{n}} \int e^{-i(x-y) \cdot(\xi-\eta)} t(x, \eta) u(y, \xi) d^{D} y d^{D} \eta \\
& \sim \sum_{I} \frac{1}{|I| !} \partial_{\xi}^{I} t D_{I, x} u
\end{aligned}
$$

where $\sim$ means up to exponentially small corrections. As one might expect, the product of an operator in $\mathcal{S}^{m}$ with an operator in $\mathcal{S}^{n}$ will be an operator in $\mathcal{S}^{m+n}$. Thus the inverse of an operator in $\mathcal{S}^{n}$ can be a PDO in $\mathcal{S}^{-n}$, and one can define groups of PDOs.

This suggests that we deal with the problem of singular perturbations which we raised at the beginning of the section, by restricting $\mathcal{O}[t]$ to be in a particular class of operators $\mathcal{S}^{m}$, with $m=2$ for the standard theory obtained by perturbing the Laplacian. Since we can use non-polynomial symbols, we can still use a family of operators as general as Eq. (20), in principle more general since the symbols need not be analytic.

Another style of definition, which would fit better with Eq. (45), would be to choose a reference operator $O[0]$, say the Laplacian, and consider the family of operators

$$
\exp t^{i} \mathcal{O}_{i} \cdot \mathcal{O}[0] ; \quad \mathcal{O}^{i} \in \mathcal{S}^{0}
$$


Either way, we are led to another question: while the physics duality conjecture is made for $m=2$; does it make sense for other $m$ ? Presumably, the relevant background solution of gravity for $m \neq 2$ would not be $\operatorname{AdS}_{D+1}$, but something else.

On the other hand, the Laplacian does seem to play a distinguished role. As discussed in $\S 3.1$, the gauge algebra in $D+1$-dimensional higher spin gravity appears to be the symmetry algebra of the Laplacian on $\mathbb{R}^{D}$, which is a small subalgebra of the algebra of differential operators. The corresponding gauge group would be functions from $M$ to the corresponding symmetry group. While this group and the group of invertible elements of $\mathcal{S}^{0}$ can both be written formally in terms of star products, the relation between them is not immediately obvious.

4.4. Multiplicative anomaly. A well studied example of Eq. (52) is to take $\mathcal{O}[0]$ to be a $2 \mathrm{~d}$ Laplacian, and $t^{i} \mathcal{O}_{i}$ to be a general function $\sigma$, in other words the family of operators

$$
O[\sigma]=e^{2 \sigma}\left(-\frac{\partial^{2}}{\partial z \partial \bar{z}}\right)
$$

In $D=2$, there is a well-known formula $[\mathbf{2}, \mathbf{4 8}]$ for the variation of $\operatorname{det} \Delta_{\gamma}$ with respect to the conformal factor $\sigma$,

$$
\delta\left(-\log \operatorname{det} \Delta_{\gamma}\right)=\frac{1}{6 \pi} \int_{\partial M} d^{2} z \sqrt{\gamma_{0}}\left(-\Delta_{\gamma_{0}} \sigma+R\left[\gamma_{0}\right]\right) \delta \sigma,
$$

where $R[\gamma]$ is the curvature scalar. While the scalar Laplacian is conformally invariant in $D=2$, the regulator needed to define the determinant is not, leading to an anomaly. However the variation is a local functional of the background curvature and conformal factor. It can be integrated to define the Liouville action Eq. (65), a functional on metrics within a given conformal class.

To directly apply the finite dimensional formulas of $\S 4.1$, we would presumably want to have a relation such as

$$
\operatorname{det} A \cdot B=\operatorname{det} A \cdot \operatorname{det} B \text {. }
$$

Of course, this is false for these operators. But there are corrected versions of these relations $[\mathbf{4 0}, \mathbf{4 6}]$ which take regularization into account, and can lead to anomalies such as Eq. (54). The basic idea is that the functional

$$
F(A, B)=\log \operatorname{det} A \cdot B-\log \operatorname{det} A-\log \operatorname{det} B
$$

is local, because its second variation $\delta_{1} \delta_{2} F(A, B)$ can be computed along the lines of Eq. (27), and vanishes for variations with disjoint support. Thus one can get a finite series expansion for it, in terms of local functionals of the coefficients of $A$ and $B$. In fact it can be expressed as a residue trace [67], a 
natural trace on the pseudo-differential operators which (in favorable cases) can be computed purely from the symbol of the operator, and agrees with the $\log$ trace.

The Liouville action was computed this way from Eq. (53) in Appendix A of [46]. In principle, the formulas there could be used to compute the analog of Eq. (54) for an arbitrary operator $\mathcal{O}[0]$ in any dimension $D$. More generally, since the relation Eq. (55) holds up to a computable local correction, one could try to follow the approach of $\$ 4.1$ making this single modification.

4.5. Relation to the RG. The usual physics intuition is that the extra dimension is related to renormalization group flow. We can see whether this has any analog in the toy model, by looking at the following simplified version of the RG. One of the standard formulations of the RG is to cut off the functional integral, in other words replace an integral over 'all' modes of a field $\phi(x)$, with an integral only over 'long wavelength' modes, say those satisfying $\Delta \psi<\Lambda^{2} \psi$ for some cutoff $\Lambda$. One then derives a formula for the variation of the functional integral with respect to $\Lambda$.

Let us consider a decomposition of the configuration space into orthonormal subspaces

$$
\mathcal{C}=\bigcup_{\lambda} \mathcal{C}_{\lambda}
$$

with projectors $d P(\lambda)$ onto $\mathcal{C}_{\lambda}$ satisfying

$$
\begin{aligned}
\mathbf{1} & =\int d P(\lambda) \\
& =\int^{\Lambda} d P(\lambda)+\int_{\Lambda} d P(\lambda) .
\end{aligned}
$$

At fixed $\Lambda$, this defines a splitting of $\mathcal{C}$ into the $\lambda<\Lambda$ and $\lambda>\Lambda$ subspaces. Parameterize these subspaces as $u$ and $v$, then we could integrate over the $\lambda>\Lambda$ subspace using a formula like

$$
\begin{array}{r}
\log \int\left[d^{2} v\right] \exp -\left(u^{\dagger} v^{\dagger}\right)\left(\begin{array}{ll}
A & B \\
C & D
\end{array}\right)\left(\begin{array}{l}
u \\
v
\end{array}\right) \\
=\operatorname{Tr} \log D+u^{\dagger}\left(A+B \cdot D^{-1} \cdot C\right) u
\end{array}
$$

to get an expression for the result of integrating out the modes $v$, as a partition function and a 'RG transformed' action $A+B \cdot D^{-1} \cdot C$. Differentiating this with respect to $\Lambda$ will define an RG flow.

In fact, we can look at Eq. (47) as a formally simpler way to accomplish the same thing, in which the variation of the cutoff is determined by the operator $\Pi\left(r^{\prime}\right)$. In this picture, we have some freedom in the specific choice of $\Pi\left(r^{\prime}\right)$ at each $r^{\prime}$, subject to the overall constraint that the evolution reach $g=1$ at the wall, corresponding to a freedom in how we vary the cutoff. 
Let us compare this $D=0$ discussion, with the RG in QFT. One very important difference is that since $\mathcal{C}$ is now infinite dimensional, we can choose isomorphisms between the subspaces $\mathcal{C}_{\lambda<\Lambda}$ for different $\Lambda$. This is what is done in the standard RG, by the device of rescaling space-time after integrating over $\mathcal{C}_{\Lambda}$. Of course, the operation of rescaling is only canonically defined for very special metrics; more generally one needs to accept that these isomorphisms are not canonical, and work with this ambiguity. This is one of the many arguments that a full understanding of the RG must be more geometrical than the existing discussions. Perhaps the gauge group in the dual higher spin gravity is related to this ambiguity; one might even conjecture that the Chern-Simons higher spin gravity of $\S 3.1$ is related to a multiplicative anomaly in the symmetry algebra of the Laplacian also discussed there.

Leaving these ideas for future work, we recall the exact renormalization group equation near a Gaussian fixed point [45],

$$
\delta_{\Lambda} e^{-S[\phi]}=\int d^{D} x d^{D} y C(x, y) \frac{\delta^{2}}{\delta \phi(x) \delta \phi(y)} e^{-S[\phi]}
$$

where

$$
S[\phi]=\int d^{D} x(\partial \phi(x))^{2}-V(\phi(x))+\cdots
$$

is an action containing all possible operators, and $\delta C(x, y)$ is the variation of the covariance with respect to the cutoff $\Lambda$.

Conceptually, this is an infinite-dimensional heat equation, and the mathematical treatments justify this picture. Thus, renormalization group evolution smooths out the integration measure $e^{-S}$, and corresponds to parabolic evolution. This looks quite different from the elliptic or hyperbolic Einstein equation, and the problem of how to reconcile these two pictures has been around since the matrix model days. Perhaps the very recent $[\mathbf{3 5}]$ will shed light on this point.

For the $O(N)$ model and free QFT, one can make a complete analysis of Eq. (62), and an attempt to derive gauge-gravity duality from this starting point will appear in $[\mathbf{2 4}]$.

\section{Chern-Simons gravity and generalized Liouville theory}

The proposed duality of free QFT and the $O(N)$ model to higher spin gravity should give us some concrete answers to the questions of the previous section. It generalizes a fairly well-studied relation which we now review.

5.1. Liouville and Chern-Simons gravity. We begin by reconsidering the simplest case of Eq. (14), the determinant of the $D=2$ scalar Laplacian with metric $\gamma$ and $t=0$, on a cylinder $\partial M \cong \mathbb{R} \times S^{1}$. For any $\gamma$, 
we can find a complex coordinate $z$, in terms of which

$$
\gamma=e^{2 \sigma} \gamma_{0} \equiv e^{2 \sigma}|d z|^{2} .
$$

As discussed in $\S 4$, the log determinant is then given by the Liouville action

$$
S_{L}=-\frac{1}{24 \pi} \int_{\partial M} d^{2} z\left[|\partial \sigma|^{2}+\lambda^{2} e^{2 \sigma}\right] .
$$

More generally, one can consider varying the conformal class of metric. In a fixed complex coordinate $z$, this could be expressed in terms of a Beltrami differential

$$
\gamma=e^{2 \sigma}|d z+\mu d \bar{z}|^{2} .
$$

Variations of $\log \operatorname{det} \Delta_{\gamma}$ with respect to $\mu$ then define correlation functions, Eq. (29) with $\mathcal{O}=\gamma^{z z} \partial_{z}^{2}$.

All of these results can be obtained from $S L(2, \mathbb{R}) \times S L(2, \mathbb{R})$ ChernSimons theory in an $\mathrm{AdS}_{3}$ background. This goes back to the 1980's work on $2 \mathrm{~d}$ quantum gravity [39], and was related to $2+1$ Chern-Simons gravity in [17]. The point that this relation is a simple version of AdS/CFT has been made in many works such as $[43,55]$.

The problem is to calculate the value of the Chern-Simons action on a flat connection $(A, \tilde{A})$ with specified boundary conditions. In general terms, this can be reduced to a calculation on $\partial M$ by writing

$$
A=g^{-1} \cdot d g ; \quad \tilde{A}=d \tilde{g} \cdot \tilde{g}^{-1}
$$

and expressing the action as a total derivative in $r$. This leads to a chiral Wess-Zumino-Witten action on $\partial M$ for each of the $S L(2, \mathbb{R})$ factors, and after taking boundary conditions corresponding to Eq. (64), to the Liouville action $[\mathbf{7}, \mathbf{1 7}]$.

One of the difficulties in carrying this out explicitly is that whereas some components of $(A, \tilde{A})$ correspond to the metric on $\partial M$, others must be determined by solving the flatness conditions. These can be simplified by a clever parameterization of $g, \tilde{g}$. Another subtlety is the need to add boundary terms to Eq. (22) which correspond to the choice of boundary conditions. To explain, the variational equations for a first order action

$$
\int_{M} A \wedge d B
$$

will include boundary terms unless we take variations with $\left.A\right|_{\partial M}=0$ (Dirichlet) or $\left.\left(\iota_{n} d B\right)\right|_{\partial M}=0$ (Neumann; here $n$ is a normal to $\left.\partial M\right)$. We can choose the other boundary conditions (say, Dirichlet for $B$ ) at the cost of adding a boundary term $\int_{\partial M} A B$.

Following $[\mathbf{7}, \mathbf{1 7}]$, one can write the flat connections Eq. (21) in the form

$$
A=r^{J_{3}}(d+\alpha) r^{-J_{3}} ; \quad \tilde{A}=r^{-J_{3}}(d+\tilde{\alpha}) r^{J_{3}},
$$


where $\alpha$ and $\tilde{\alpha}$ are flat $S L(2, \mathbb{R})$ connections on a surface of fixed $r$, and $\left(J^{+}, J^{3}, J^{-}\right)$is a basis of the Lie algebra $s l(2, \mathbb{R})$. Taking the surface at $r \rightarrow 0$, we can use $(\alpha, \tilde{\alpha})$ to specify the metric (actually the frame or zweibein) on $\partial M$.

Expanding Eq. (69) gives us

$$
\begin{aligned}
& A=\frac{1}{r} \alpha^{-} J_{-}+\left(-\frac{d r}{r}+\alpha^{3}\right) J^{3}+r \alpha^{+} J_{+} \\
& \tilde{A}=\frac{1}{r} \tilde{\alpha}^{+} J_{+}+\left(\frac{d r}{r}+\tilde{\alpha}^{3}\right) J^{3}+r \tilde{\alpha}^{-} J_{-},
\end{aligned}
$$

so they correspond to a frame and metric on $\partial M$

$$
\begin{aligned}
e & =\frac{1}{2}\left(\alpha^{-} J_{-}-\tilde{\alpha}^{+} J_{+}\right) \\
d s^{2} & =\frac{1}{r^{2}}\left(d r^{2}-\alpha^{-} \tilde{\alpha}^{+}\right)+\frac{d r}{r} \cdot\left(\alpha^{3}-\tilde{\alpha}^{3}\right)+\mathcal{O}\left(r^{2}\right) .
\end{aligned}
$$

The cross term $d r \cdot \alpha$ does not belong in an expansion of the form Eq. (11), but it can be canceled by taking a connection with $\alpha^{3}=\tilde{\alpha}^{3}$. This implicitly tells us what type of boundary terms must be added to Eq. (22); see [7] for the details.

Evaluating the resulting action on Eq. (69), one finds

$$
\begin{aligned}
S_{S G}= & -\frac{1}{3} \int_{M} \operatorname{Tr}\left[(\alpha)^{3}-(\tilde{\alpha})^{3}+3\left(\left(\alpha^{2}+\tilde{\alpha}^{2}\right) J_{3} d r\right]\right. \\
& +\int_{\partial M} r^{-2} \alpha^{-} \tilde{\alpha}^{+}-\frac{1}{2} \alpha^{3} \tilde{\alpha}^{3}+r^{2} \alpha^{+} \tilde{\alpha}^{-} .
\end{aligned}
$$

This contains a quadratically divergent term $r^{-2} \int_{\partial M} \alpha^{-} \tilde{\alpha}^{+}$, and a logarithmically divergent term $\int d r \ldots$ Both are expected divergences in the QFT, the first proportional to the volume, and the second reproducing the trace anomaly.

The rest of the action is finite, and reproduces the Liouville action Eq. (65) (shown in $[\mathbf{7}, \mathbf{1 7}]$ ), as well as the generating functional of stresstensor correlators defined using the metric Eq. (66) (shown in [7]). Combining this with the physics derivations of these results for the $2 \mathrm{~d}$ free boson, or the more general approach of $\S 4.4$, we have explained 'Fact 1' of the introduction.

5.2. Generalized Liouville theory. We can now explain 'Conjecture 1 ' of the introduction. It is a generalization of the above, starting from higher spin Chern-Simons gravity, fixing boundary conditions to obtain a generalization of the $S L(2, \mathbb{R})$ Wess-Zumino-Witten or Liouville actions. The duality relation Eq. (3) then states that this is equal to the logarithm of the 
determinant of a general 2d linear pseudo-differential operator. Similar conjectures should hold in any dimension $D$ with a higher spin gravity theory, as in $D=3$ where it follows from the physics conjecture of $[\mathbf{3 8}, \mathbf{5 3}]$.

Although the conceptual parallel with the $S L(2, \mathbb{R}) \times S L(2, \mathbb{R})$ analysis seems clear, we have not yet worked out the details. Some steps in this direction are taken in $[\mathbf{1 5}, \mathbf{3 6}]$. The starting point is to generalize Eq. (69), and identify the leading coefficients in the expansion of $\alpha, \tilde{\alpha}$ in powers of $r$, as coefficients in the expansion Eq. (20). Thus we identify the generators of $S L(2, \mathbb{R}) \subset h s(1,1)$ as in Eq. (26), and generalize Eq. (70) to

$$
\begin{aligned}
A= & -\frac{d r}{r} \xi^{1} \xi^{2}+\frac{1}{r}\left(e^{\sigma} d z \xi^{1} \xi^{1}+\sqrt{\gamma^{(4)}} \xi^{1} \xi^{1} \xi^{1} \xi^{1}+\sqrt{\gamma^{(6)}}\left(\xi^{1}\right)^{6}+\cdots\right) \\
& +\mathcal{O}\left(r^{0}\right)
\end{aligned}
$$

(resp. $\tilde{A}$ ), where the subleading terms are determined by the flatness conditions, perhaps using a generalization of the Bruhat decomposition of $A=$ $g^{-1} d g$ used in [7].

Here we have written $\gamma^{(2)}=e^{2 \sigma}$ and generalized the higher order coefficients in like fashion. Of course, there are many further components of the tensors $\gamma^{(k)}$ in Eq. (20), but as these can be simplified into terms involving the Laplacian, they only lead to local effects.

We should distinguish the $2 \mathrm{~d}$ conjecture under discussion from another statement about determinants. This is based on the well known physics relation between $2 \mathrm{~d}$ free QFTs (bosons or fermions) coupled to gauge connections, and Wess-Zumino-Witten theory [63]. For free fermions, this states that

$$
\operatorname{Tr} \log D_{\mathcal{A}}=S_{W Z W}(G)+\int_{\partial M} \operatorname{tr} \mathcal{A} \cdot G^{-1} d G
$$

where $D_{\mathcal{A}}$ is a connection on a rank $K$ vector bundle $E$ over $\partial M, G$ is a map $\partial M \rightarrow G L(K)$, and we evaluate the right hand side at a critical point in $G$. This can also be expressed in terms of the Chern-Simons action $[\mathbf{2 6}]$, and is another example of the relation between anomalies (here in chiral $G L(K)$ invariance) and Chern-Simons.

Given this relation, and then relating higher order differential operators to systems of first order operators as

$$
\operatorname{det}\left(\sum_{k}^{K} \gamma^{(k)} \partial^{k}\right) \leftrightarrow \operatorname{det}(\partial \cdot \mathbf{1}+\mathcal{A}),
$$

one gets an alternate 'holographic' description of these determinants, in terms of a gauge theory with a finite number of fields. However, no analog of this second description is known for dimensions $D>2$. Perhaps it would be worth searching for one, but physicists have looked hard for bosonization 
formulas in $D>2$, with limited success. The present conjecture is quite different in using an infinite number of fields.

If Conjecture 1 holds, as do analogous conjectures in other dimensions $D$, since the picture is so general, it seems very likely to us that it will have a simple proof, perhaps along the lines sketched in section 4 .

\section{References}

[1] O. Aharony, S. S. Gubser, J. M. Maldacena, H. Ooguri and Y. Oz, "Large N field theories, string theory and gravity," Phys. Rept. 323, 183 (2000) [arXiv:hepth/9905111].

[2] O. Alvarez, "Theory Of Strings With Boundaries: Fluctuations, Topology, And Quantum Geometry," Nucl. Phys. B 216, 125 (1983).

[3] M. T. Anderson, in AdS/CFT Correspondence: Einstein metrics and their conformal boundaries; 73rd Meeting of Theoretical Physicists and Mathematicians, Strasbourg, September 11-13, 2003, ed. O. Biquard, European Mathematical Pub. House, (2005) [arXiv:hep-th/0403087].

[4] A. Ashtekar, "New Variables for Classical and Quantum Gravity," Phys. Rev. Lett. $\mathbf{5 7}, 2244$ (1986).

[5] S. Axelrod and I. M. Singer, "Chern-Simons perturbation theory," arXiv:hepth/9110056.

[6] S. Axelrod and I. M. Singer, "Chern-Simons perturbation theory. 2," J. Diff. Geom. 39, 173 (1994) [arXiv:hep-th/9304087].

[7] M. Banados, O. Chandia and A. Ritz, "Holography and the Polyakov action," Phys. Rev. D 65, 126008 (2002) [arXiv:hep-th/0203021].

[8] T. Banks, M. R. Douglas and N. Seiberg, "Probing F-theory with branes," Phys. Lett. B 387, 278 (1996) [arXiv:hep-th/9605199].

[9] N. Beisert, B. Eden and M. Staudacher, "Transcendentality and crossing," J. Stat. Mech. 0701, P021 (2007) [arXiv:hep-th/0610251].

[10] M. P. Blencowe, "A Consistent Interacting Massless Higher Spin Field Theory In D $=(2+1)$, , Class. Quant. Grav. 6, 443 (1989).

[11] E. Bergshoeff, M. P. Blencowe and K. S. Stelle, "Area Preserving Diffeomorphisms And Higher Spin Algebra," Commun. Math. Phys. 128, 213 (1990).

[12] E. Brezin, C. Itzykson, G. Parisi and J. B. Zuber, "Planar Diagrams," Commun. Math. Phys. 59, 35 (1978).

[13] E. Brezin and V. A. Kazakov, "Exactly Solvable Field Theories of Closed Strings," Phys. Lett. B 236, 144 (1990).

[14] J. D. Brown and M. Henneaux, "Central Charges in the Canonical Realization of Asymptotic Symmetries: An Example from Three-Dimensional Gravity," Commun. Math. Phys. 104, 207 (1986).

[15] A. Campoleoni, S. Fredenhagen, S. Pfenninger and S. Theisen, "Asymptotic symmetries of three-dimensional gravity coupled to higher-spin fields," arXiv:1008.4744 [hep-th].

[16] S. R. Coleman, "Quantum sine-Gordon equation as the massive Thirring model," Phys. Rev. D 11, 2088 (1975).

[17] O. Coussaert, M. Henneaux and P. van Driel, "The Asymptotic dynamics of threedimensional Einstein gravity with a negative cosmological constant," Class. Quant. Grav. 12, 2961 (1995) [arXiv:gr-qc/9506019].

[18] J. de Boer, E. P. Verlinde and H. L. Verlinde, "On the holographic renormalization group," JHEP 0008, 003 (2000) [arXiv:hep-th/9912012].

[19] S. R. Das and A. Jevicki, "String field theory and physical interpretation of D = 1 strings," Mod. Phys. Lett. A 5, 1639 (1990). 
[20] S. R. Das and A. Jevicki, "Large-N collective fields and holography," Phys. Rev. D 68, 044011 (2003) [arXiv:hep-th/0304093].

[21] S. Deser, "Self-interaction and gauge invariance," Gen. Rel. Grav. 1, 9 (1970) [arXiv:gr-qc/0411023].

[22] P. Di Francesco, P. H. Ginsparg and J. Zinn-Justin, "2-D Gravity and random matrices," Phys. Rept. 254, 1 (1995) [arXiv:hep-th/9306153].

[23] M. R. Douglas and S. H. Shenker, "Strings In Less Than One-Dimension," Nucl. Phys. B 335, 635 (1990).

[24] M. R. Douglas, L. Mazzucato and S. S. Razamat, "Holographic dual of free field theory," Phys. Rev. D83, 071701 (2011) [arXiv:1011.4926 [hep-th]].

[25] M. G. Eastwood, "Higher symmetries of the Laplacian," Annals Math. 161, 1645 (2005) [arXiv:hep-th/0206233].

[26] S. Elitzur, G. W. Moore, A. Schwimmer and N. Seiberg, "Remarks On The Canonical Quantization Of The Chern-Simons-Witten Theory," Nucl. Phys. B 326, 108 (1989).

[27] C. Fefferman and C.R. Graham, "Conformal invariants," in: Élie Cartan et les Mathematiques dAujourdhui, Astérisque, 1985, Numero hors serie, Soc. Math. France, Paris, pp. 95-116.

[28] S. Giombi and X. Yin, "Higher Spins in AdS and Twistorial Holography," arXiv:1004.3736 [hep-th].

[29] R. Gopakumar, "Free field theory as a string theory?," Comptes Rendus Physique 5, 1111 (2004) [arXiv:hep-th/0409233].

[30] M. B. Green, J. A. Harvey and G. W. Moore, "I-brane inflow and anomalous couplings on D-branes," Class. Quant. Grav. 14, 47 (1997) [arXiv:hep-th/9605033].

[31] N. Gromov, V. Kazakov and P. Vieira, "Exact Spectrum of Planar $\mathcal{N}=4$ Supersymmetric Yang-Mills Theory: Konishi Dimension at Any Coupling," Phys. Rev. Lett. 104, 211601 (2010) [arXiv:0906.4240 [hep-th]].

[32] D. J. Gross and A. A. Migdal, "Nonperturbative Two-Dimensional Quantum Gravity," Phys. Rev. Lett. 64, 127 (1990).

[33] S. S. Gubser, I. R. Klebanov and A. M. Polyakov, "Gauge theory correlators from non-critical string theory," Phys. Lett. B 428, 105 (1998) [arXiv:hep-th/9802109].

[34] A. Guionnet, "Large deviations and stochastic calculus for large random matrices," Probability Surveys 1, 72-172 (2004) [arXiv:math/0409277].

[35] I. Heemskerk and J. Polchinski, "Holographic and Wilsonian Renormalization Groups," arXiv:1010.1264 [hep-th].

[36] M. Henneaux and S. J. Rey, "Nonlinear W(infinity) Algebra as Asymptotic Symmetry of Three-Dimensional Higher Spin Anti-de Sitter Gravity," arXiv:1008.4579 [hep-th].

[37] M. Henningson and K. Skenderis, "The holographic Weyl anomaly," JHEP 9807, 023 (1998) [arXiv:hep-th/9806087].

[38] I. R. Klebanov and A. M. Polyakov, "AdS dual of the critical O(N) vector model," Phys. Lett. B 550, 213 (2002) [arXiv:hep-th/0210114].

[39] V. G. Knizhnik, A. M. Polyakov and A. B. Zamolodchikov, "Fractal structure of 2d-quantum gravity," Mod. Phys. Lett. A 3, 819 (1988).

[40] M. Kontsevich and S. Vishik, "Geometry of determinants of elliptic operators," [arXiv: hep-th/9406140].

[41] J. M. Maldacena, "The large N limit of superconformal field theories and supergravity," Adv. Theor. Math. Phys. 2, 231 (1998) [Int. J. Theor. Phys. 38, 1113 (1999)] [arXiv:hep-th/9711200].

[42] D. Martelli, J. Sparks and S. T. Yau, "The geometric dual of a-maximisation for toric Sasaki-Einstein manifolds," Commun. Math. Phys. 268, 39 (2006) [arXiv:hepth/0503183]. 
[43] E. J. Martinec, "Conformal field theory, geometry, and entropy," arXiv:hepth/9809021.

[44] J. A. Minahan and K. Zarembo, "The Bethe-ansatz for N = 4 super Yang-Mills," JHEP 0303, 013 (2003) [arXiv:hep-th/0212208].

[45] P. K. Mitter, "The exact renormalization group," contribution to the Encyclopedia of Mathematical Physics, Elsevier 2006; arXiv:math-ph/0505008.

[46] K. Okikiolu, "The multiplicative anomaly for determinants of elliptic operators," Duke Math. J. 79 (3), 723-750 (1995).

[47] J. Polchinski, "Classical Limit Of (1+1)-Dimensional String Theory," Nucl. Phys. B 362, 125 (1991).

[48] A. M. Polyakov, "Quantum geometry of Bosonic strings," Phys. Lett. B 103, 207 (1981).

[49] A. M. Polyakov, Gauge Fields and Strings, Harcourt, 1987.

[50] A. M. Polyakov, "String theory and quark confinement," Nucl. Phys. Proc. Suppl. 68, 1 (1998) [arXiv:hep-th/9711002].

[51] D. B. Ray and I. M. Singer, Adv. Math., 7:145, 1971.

[52] X. Saint Raymond, Elementary Introduction to the Theory of Pseudodifferential Operators, CRC Press, 1991.

[53] E. Sezgin and P. Sundell, "Massless higher spins and holography," Nucl. Phys. B 644, 303 (2002) [Erratum-ibid. B 660, 403 (2003)] [arXiv:hep-th/0205131].

[54] I. M. Singer, "On the Master Field in Two Dimensions," in Progress in Mathematics, vol. 131, pp. 263-282, Birkhauser, 1995.

[55] K. Skenderis and S. N. Solodukhin, "Quantum effective action from the AdS/CFT correspondence," Phys. Lett. B 472, 316 (2000) [arXiv:hep-th/9910023].

[56] D. T. Son, "AdS/CFT overview and recent applications to strongly interacting systems," Nucl. Phys. A 827, 61C (2009).

[57] L. B. Szabados, "Quasi-local energy-momentum and angular momentum in GR: A review article," Living Rev. Rel. 7 (2004) 4.

[58] G. 't Hooft, Fifty years of Yang-Mills theory, World Scientific, 2005.

[59] M. A. Vasiliev, "Higher-spin gauge theories in four, three and two dimensions," Int. J. Mod. Phys. D 5, 763 (1996) [arXiv:hep-th/9611024].

[60] M. A. Vasiliev, "Higher spin gauge theories in any dimension," Comptes Rendus Physique 5, 1101 (2004) [arXiv:hep-th/0409260].

[61] D. V. Voiculescu, "Free Entropy," [arXiv:math/0103168v1].

[62] D. V. Voiculescu, K. J. Dykema and A. Nica, Free Random Variables, AMS, 1992.

[63] E. Witten, "Nonabelian bosonization in two dimensions," Commun. Math. Phys. 92, 455 (1984).

[64] E. Witten, "(2+1)-Dimensional Gravity as an Exactly Soluble System," Nucl. Phys. B 311, 46 (1988).

[65] E. Witten, "Anti-de Sitter space and holography," Adv. Theor. Math. Phys. 2, 253 (1998) [arXiv:hep-th/9802150].

[66] E. Witten and S. T. Yau, "Connectedness of the boundary in the AdS/CFT correspondence," Adv. Theor. Math. Phys. 3, 1635 (1999) [arXiv:hep-th/9910245].

[67] M. Wodzicki, "Noncommutative residue. I. Fundamentals, K-theory, arithmetic and geometry," in Lecture Notes in Math., vol. 1289, Springer, Berlin, 1987, pp. 320399. 
